\title{
Atomic Scale Manipulation of Grain Boundary Structures Through Doping and in-situ Gas Reduction
}

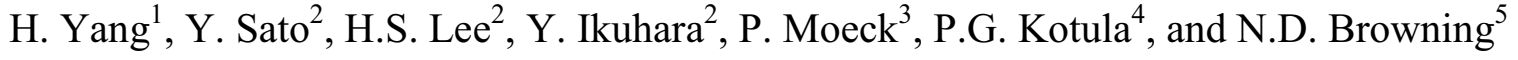 \\ 1 University of California-Davis, One Shields Avenue, Davis, CA 95616, USA \\ 2 The University of Tokyo, 2-11-16, Yayoi, Bunkyo, Tokyo 113-8656, Japan \\ 3 Portland State University, Portland OR 97201-0751, USA \\ 4 Sandia National Laboratories, Albuquerque NM 87185, USA \\ 5 Pacific Northwest National Laboratory, Richland, WA 99352, USA
}

Grain boundaries (GBs) are known to have far-reaching effects on the electrical and mechanical properties of materials. Understanding the atomic scale mechanisms behind these effects requires an accurate determination of the interplay between GB structure and composition. Based on the analysis of a range of grain boundaries using aberration corrected scanning transmission electron microscopy (STEM), a general structural units model has been derived for the structure of grain boundaries in various dense packing cubic materials including FCC metals, perovskites, fluorites etc. (Fig.1) [1] The similarities in the observed grain boundary structures of these materials originate from related space (and point) group symmetries of the parent structures (Fig.2). The presence of structural variations away from the general structural units model may be caused by frustrations of certain symmetry operations that result from the incorporation of point defects (vacancies and impurities). A clear understanding of the similarity and variation in grain boundary atomic structures will not only provide a means to infer the structure-property relationships in broad classes of materials, but also enables us eventually to effectively manipulate the GB structures to achieve better materials properties.

To understand these chemical induced variations, and further quantify exactly how atomic scale variations at the boundary plane extend to the practical mesoscale operating length of the system, statistical analysis has been applied to the aberration corrected STEM Z-contrast images acquired from a series of undoped [2] and doped $\mathrm{SrTiO}_{3}$ GBs. In order to understand the effects of oxygen vacancies incorporation, in-situ characterization of GB atomic structures were performed using the aberration corrected FEI Environmental TEM under the reduced gas and heating environment. The reduction rate is further quantified as a function of the number of oxygen vacancies generated during the reduction process. This analysis of GB similarity and variation provides insights into the structure-composition relationship in GBs. It also helps to determine experimentally the energetics behind the formation of grain boundary structures to predict GB formation in various materials. [3]

\section{References:}

[1] Tong, W., et al., Acta Mater. (In Press).

[2] Yang, H., et al., Philos. Mag. 1 (2012).

[3] Acknowledgments: This work is supported by the United States Department of Energy Grant No. DE-FG02-03ER46057. The authors thank the support from EMSL, a national scientific user facility located at Pacific Northwest National Laboratory, which is operated by Battelle for the U.S.Department of Energy under contract DE-AC05-76RL01830. 

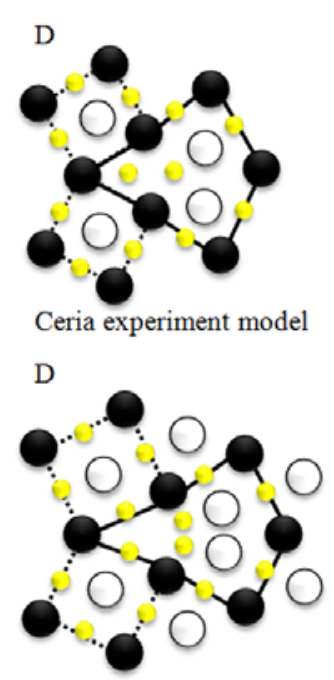

YSZ: $36^{\circ} \sum 5 \mathrm{~GB}$ CSL model

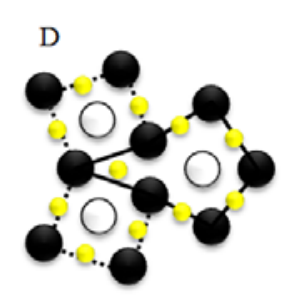

$\mathrm{SrTiO}_{3}$ experiment model

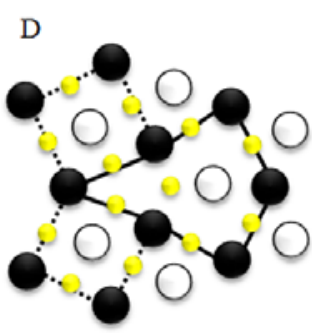

YSZ: $36^{\circ} \sum 5 \mathrm{~GB}$ experiment model

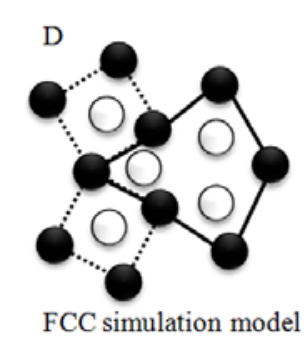

A'

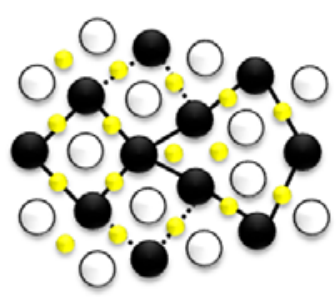

YSZ: $24^{\circ}$ near $-\sum 13 \mathrm{~GB}$ experiment model

Fig. 1. Sketches of structural units in different grain boundaries including $\mathrm{CeO}_{2}, \mathrm{SrTiO}_{3}$, Face Centred Cubic (FCC) metals, and Yttrium Stabilized Zirconia (YSZ). Structural units share a general schema with corresponding atomic columns located at identical positions despite the differences in crystal structure and chemical composition.
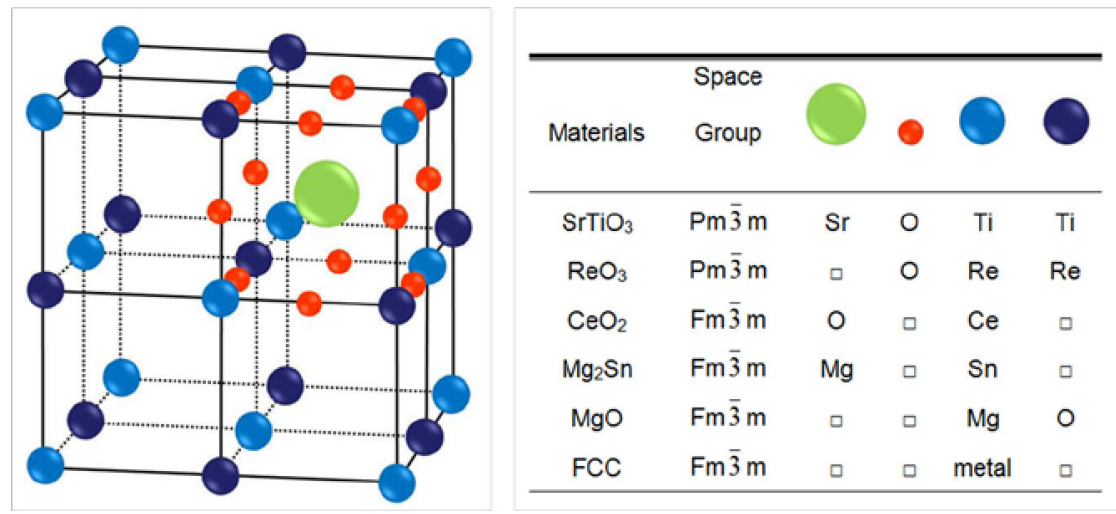

Fig. 2. Cubic perovskite and some derived structures. Spheres of a certain color represent ions in the table to the right. Only in one octant have all ions been plotted. The "Schottky void symboll" stands for unoccupied ion positions. The second column of the table gives the space groups of the crystal structures. Note that all of these structures possess point group $m \overline{3} m$. 